\title{
Immune Response to Infection with Strongyloides stercoralis in Patients with Infection and Hyperinfection
}

\author{
Jill E. Weatherhead • Rojelio Mejia
}

Published online: 20 September 2014

(C) Springer International Publishing AG 2014

\begin{abstract}
Helminthic infections are known to induce host immune responses and polarization. The ubiquitous Strongyloides stercoralis can remain relatively asymptomatic in the host for decades, until there is a change in the host immune balance. Factors that influence these changes range from immunosuppressives, including corticosteroids, to acquired viral infections, such as HTLV-1. These conditions play an important role in the progression of disease for strongyloidiasis. The alteration of the Th-1, Th-2 balance can induce hyperinfection and dissemination of these helminths, changing a minor clinical process to a life-threatening condition.
\end{abstract}

Keywords Strongyloides stercoralis · Strongyloidiasis . Th-2 $\cdot$ Immunology $\cdot$ Immunosuppression $\cdot \mathrm{HIV} \cdot \mathrm{HTLV}-1$

\section{Introduction}

Strongyloides stercoralis is an intestinal helminth that causes acute and chronic infection in humans. While the precise prevalence is unknown, strongyloidiasis has been documented throughout the world, particularly in tropical and sub-tropical areas [1]. Natural transmission of Strongyloides has been reported in most countries excluding Canada and other cold weather climates [2]. Approximately $50-100$ million people are infected with Strongyloides; however, this is likely a gross underestimate of true prevalence of disease [1-3]. In the United States, acquisition of primary infection has been

J. E. Weatherhead $\cdot$ R. Mejia $(\bowtie)$

National School of Tropical Medicine, Baylor College of Medicine, 1102 Bates St. Feigin Bldg, Suite 0550.01, Houston, TX 77030, USA

e-mail: rmejia@bcm.edu

J. E. Weatherhead

e-mail: weatherh@bcm.edu documented in Southeastern and Appalachian regions with rates as high as $4 \%$ of the population being infected $[2,4]$. Among at-risk populations in endemic regions of the world, prevalence rates have been reported to be as high as $12-26 \%$ $[1,5]$. Alterations in the host immune system as a result of infection with HTLV-1, immunosuppressant medications, malignancies, organ transplant, chronic malnutrition, and other chronic disease such as diabetes mellitus, chronic obstructive pulmonary disease, alcohol use, and chronic renal failure have been associated with increased rates of infection as well as more serious disease $[3,6]$.

Strongyloides has a unique life cycle that allows completion of the entire cycle within the human host through autoinfection $[1,5]$. On initial exposure, filariform larvae infect the human host transcutaneously. Larvae subsequently migrate via venous blood through the lungs, ascend the tracheobronchial tree and complete their life cycle in the host duodenum [6]. During acute larvae migration, patients may present with pulmonary symptoms including cough, wheezing, hemoptysis, and may have interstitial infiltrates seen on imaging or gastrointestinal symptoms including crampy abdominal pain, diarrhea, anorexia, vomiting, and rarely ileus or small bowel obstruction $[4,6]$. After initial infection, mature females in the duodenum produce rhabidtiform larvae through parthenogenesis. Rhabidtiform larvae in the intestinal tract may develop into infective filariform larvae and re-infect the host through penetration of the rectal tissue, a process called autoinfection [5]. Autoinfection cycles can continue for decades creating a chronic carrier state of strongyloidiasis in the human host [1, 5]. Patients with chronic strongyloidiasis are typically asymptomatic, but may have intermittent eosinophilia during times of larvae migration through tissue with a distinctive dermal erythematous tract called larva currens [3, 6], or minor intermittent gastrointestinal symptoms such as diarrhea, vomiting, or constipation over time [1, 6, 7]. Hosts can live with chronic strongyloidiasis, acquired from 
remote exposure, for decades without significant evidence of infection [1,5].

Despite the relative asymptomatic state of chronic disease, strongyloidiasis can progress to more serious infection in the event of alteration of the host immune response [8•]. Decreased immune surveillance in the gastrointestinal mucosa secondary to immunosuppression permits acceleration of the autoinfection cycle [4]. Increased production of larvae in the duodenum leads to increased larvae migration through the gastrointestinal mucosa and through the lungs creating exaggerated gastrointestinal and pulmonary symptoms, termed hyperinfection syndrome $[1,3,4,6]$. Because of the large burden of larvae migration within tissue, patients can present with progressive acute respiratory failure and hemorrhagic pneumonitis as well as worsening abdominal pain, diarrhea, and possible gastrointestinal obstruction $[1,3]$. Increased larvae migration across the gastrointestinal mucosa creates significant mucosal damage allowing for translocation of enteric gram-negative bacteria into the blood stream [6]. Patients with Strongyloides hyperinfection may present with gram-negative bacteria sepsis, meningitis, and pneumonia contributing to significant morbidity and mortality [3, 6, 9]. Unlike in chronic strongyloidiasis, patients with hyperinfection syndrome typically do not have peripheral eosinophilia [3].

Disseminated disease refers to larvae that are found in organs not involved in the standard lifecycle of Strongyloides [6]. Disseminated strongyloidiasis has been documented to involve the meninges and other areas of the central nervous system, the biliary tract, pancreas, and liver [1]. Both hyperinfection syndrome and disseminated disease are associated with case fatality rates greater than $50 \%$ and reaching up to $70-85 \%$ in certain high-risk populations $[3,10]$.

\section{Strongyloides and the Host Immune System}

The host innate and adaptive immune response plays a critical role in the maintenance of chronic strongyloidiasis and the prevention of hyperinfection syndrome and dissemination. Similar to other helminth infections, strongyloidiasis elicits a Th-2 lymphocyte predominant immune response with production of cytokines, IgE antibodies, eosinophils, and mast cells which participate in expulsion and killing of the helminth [3, 7, 11]. Strongyloides antigens activate eosinophils via the innate immune response [12]. Activated eosinophils act as antigen presenting cells to stimulate antigen-specific Th-2 cytokine production including IL-4 and IL-5 [8•, 12]. IL-4 induces activated B lymphocytes to class-switch for production of IgE and IgG4 antibodies and additional cytokines (IL8) attract granulocytes such as neutrophils to aid in larvae killing $[7,11,12]$. IgE production allows for mast cell degranulation and enhances further eosinophil migration [8•]. IL-5 acts as an eosinophil colony stimulating factor promoting further eosinophil growth and activation [8・ 11, 12]. Approximately $75 \%$ of patients with chronic strongyloidiasis have peripheral eosinophilia or elevated total $\operatorname{IgE}$ levels $[4,12]$. Protective immunity to infective larvae has been found to involve specific Strongyloides antibodies, complement activation and neutrophils in antibody-dependent, cell-mediated cyotoxicity type responses [11]. Patients with severe disease have been shown to have a significant decrease in antibody levels and a decrease in eosinophil level compared to asymptomatic infected individuals, suggesting that both antibodies and granulocytes play a significant role in protection from infection [11]. The sophisticated interaction between strongyloidiasis and the host immune system allows for long-term survival of the pathogen in the host gastrointestinal tract. However, disruption of the host immune system with loss of granulocyte function, humoral-mediated antibody production, or Th-2 cytokine release, reduces immune regulation of adult worm density and predisposes patients to hyperinfection syndrome and dissemination $[3,4,13 \cdot]$. Changes in the immune system secondary to diabetes, hematologic malignancies, malnutrition, hypogammaglobulinemia, use of immunosuppressive drugs such as corticosteroids in autoimmune disease, chronic obstructive pulmonary disease or malignancy, organ transplantation, and HTLV-1 are associated with increased risk of hyperinfection and dissemination $[1,14 \bullet]$.

\section{Human T-Lymphotropic Virus-1 (HTLV-1)}

HTLV-1 is a human RNA retrovirus endemic in areas of Japan, Caribbean, and certain locations in Africa and South America, and is the cause of HTLV-1 associated myelopathy and adult T-cell leukemia and lymphoma (ATLL) $[11,12,15]$. Similar to other retroviruses such as human immunodeficiency virus (HIV), HTLV-1 is transmitted in three different pathways, vertically via transplacental route or breast milk, horizontally through sexual contact, or parenterally via blood transfusion or intravenous drug use [5]. Unlike HIV, patients with HTLV-1 are not susceptible to opportunistic infections [12]. Furthermore, in comparison to HIV, HTLV-1 infects Tcells, induces spontaneous T-cell proliferation, promotes a hyper Th-1 immune response with production of high levels of INF-gamma, and activates regulatory T-cells to produce increased levels of IL-10 and TNF-beta [5, 7, 11, 12].

In patients co-infected with HTLV-1 and Strongyloides, a unique misbalance of the immune system occurs leading to increased susceptibility to severe helminth disease. HTLV-1, which predisposes the patient to an immunologic shift to a Th1 dominated immune response, blunts the Th- 2 response to helminth infection such as strongyloidiasis [12]. As a result of decreased Th- 2 response, there are decreased serum concentrations of IL-4, IL-5, IL-13, and specific IgE antibodies against $S$. stercoralis $[6,7,8 \cdot 12]$. A decrease in IL-4 and 
IgE subsequently reduces mast cell function, eosinophil recruitment, and the ability of the host immune system to kill the worm creating an environment primed for Strongyloides propagation [3, 11]. Patients with co-infection of Strongyloides and HTLV-1 infection have significantly lower eosinophil counts and IgE levels in the serum compared to patients with Strongyloides without co-infection with HTLV-1 [5]. The alteration in cytokine, granulocyte, and antibody production correlates with high prevalence of strongyloidiasis, a higher rate of recurrent strongyloidiasis, a higher rate of more severe disease such as hyperinfection syndrome and dissemination, and a higher rate of therapeutic failure in HTLV-1 carriers [3, $5,15]$. Specifically, eradication rate of $S$. stercoralis by ivermectin has been shown to be significantly lower in patients with HTLV-1 infection compared to those without HTLV-1 infection [5].

Co-infection of HTLV-1 and Strongyloides also has significant affects on the progression of HTLV-1 infection in the host. Strongyloides facilitates HTLV-1 virus replication allowing for higher levels of circulating virus, which have been shown to decline after treatment with ivermectin $[4,6]$. Co-infected patients tend to have increased integration of HTLV-1 pro-viral DNA in lymphocytes accelerating development of ATLL $[6,15]$. Patients with HTLV-1 and strongyloidiasis who develop ATLL typically are significantly younger than patients without strongyloidiasis who proceed to develop ATLL. Strongyloidiasis may serve as a co-factor in the development of ATLL in patients with HTLV-1 by shortening the latency period to leukomogenesis $[6,15]$.

\section{Human Immunodeficiency Virus/Acquired Immunodeficiency Syndrome (HIV/AIDS)}

Strongyloidiasis hyperinfection syndrome and strongyloidiasis dissemination was initially an AIDS defining illness. However, due to the rare incidence of hyperinfection and disseminated strongyloidiasis in patients with HIV/AIDS, the Center for Disease Control (CDC) and the World Health Organization (WHO) have subsequently removed strongyloidiasis from the list of defining infections [8•, 9]. While patients with HIV have a high risk of intestinal strongyloidiasis, patients with HIV are not at increased risk for hyperinfection or dissemination $[3,8 \cdot 11]$. In a study conducted at the National Institutes of Health (NIH), 26 out of 103 subjects with AIDS (CD4< 100) were seropositive for strongyloidiasis giving an overall prevalence of $25 \%$ within this cohort [13•]. In contrast to HTLV-1, HIV infects CD4 T lymphocytes and induces T-cell destruction [12]. As a result, the immune system sustains an acute decline in Th-1 lymphocytes in comparison to Th-2 lymphocytes. The profound loss of Th-1 with minimal change in Th-2 mediated cytokine release promotes mucosal regulation of strongyloidiasis [8•]. Furthermore, HIV has been associated with high levels of IgE, increased production of eosinophils, and suppression of the development of Strongyloides larvae in the gut required for autoinfection [8•, 9]. Despite the low likelihood of hyperinfection syndrome in patients with immunosuppression secondary to AIDS, cases of immune reconstitution inflammatory syndrome (IRIS) with initiation of anti-retroviral therapy have been described [13•]. While the pathophysiology of this process is unknown, some experts have postulated that reconstitution of the Th-1 immune response may promote development of invasive filariform larvae capable of autoinfection and migration [6,9]. While severe infection is typically not found in HIV infection, the high prevalence and possibility to induce IRIS in patients receiving anti-retroviral therapy suggest that empiric therapy with an anthelminthic agent may be justified in all AIDS patients from strongyloidiasis endemic areas $[13 \bullet]$.

\section{Steroids and Other Immunosuppressant Medications}

Progression of chronic strongyloidiasis to hyperinfection and disseminated disease has been well described with the use of immunosuppressant medications such as corticosteroids. Case reports of strongyloidiasis hyperinfection syndrome in patients with lupus, rheumatoid arthritis, pyomyositis, nephrotic syndrome, and other autoimmune diseases that are typically maintained with steroids have been documented [6]. The increased risk of hyperinfection syndrome in the setting of steroid use persists regardless of steroid dose, duration of therapy or administration route and can even occur in settings of elevated endogenous or pharmacologic adrenocorticotropin levels [4, 6]. Signs and symptoms of augmented autoinfection and hyperinfection syndrome occur as early as 20 days after the onset of steroid therapy and as late as several years post-steroid therapy [6]. Theories behind this association include the ability of glucocorticoid to suppress eosinophil proliferation, promote apoptosis of Th-2 lymphocytes, blunt mucosal mast cell response to Strongyloides antigens, accelerate transformation of rhabitiform larvae into filariform larvae, and possibly rejuvenate reproductively latent adult females into parthenogenesis $[3,6]$.

Hyperinfection syndrome has been associated with other immunosuppressive medications as well including vincristine, azathioprine, cyclophosphamide, antithymocyte globulin, anti-cd3, 6-mercaptopurine, methotrexate, bleomycin, adriamycin, ifosfamide, melphalan, CP16, total body irradiation, etanercept, infixumab, rituximab, and mycophenalate [6, $16 \bullet$. However, in many of these reports, patients also received concurrent glucocorticoids making it difficult to assess the true relationship between the immunosuppressant agent and 
Strongyloides hyperinfection. A small number of case reports have demonstrated vincristine and other vinca alkaloids may decrease intestinal motility and may facilitate development of rhabidtiform larvae into invasive filariform larvae putting the host at increased risk of autoinfection propagation [6]. Additional case reports of patients with rheumatoid arthritis on chronic low dose prednisone have developed strongyloidiasis hyperinfection after the initiation of TNF-alpha inhibitors suggesting that TNF-alpha inhibitors contribute to propagation of the autoinfection cycle of strongyloidiasis as well [17].

\section{Solid Organ Transplant and Hematopoietic Stem Cell Transplant}

Strongyloides hyperinfection syndrome and dissemination has been documented in cases of solid organ transplant including kidney, heart, lung, intestine, pancreas, and liver, as well as hematopoietic stem cell transplant [3]. Disease typically occurs in transplant patients due to activation of remotely acquired, asymptomatic strongyloidiasis in the recipient; however, allograft transmission has been documented [1]. Transplant patients are at particular risk of progression from chronic infection to hyperinfection and dissemination due to the significant role of immunosuppression. Prior to transplant, patients receive pre-conditioning with immunosuppressive therapies and post-transplant patients receive chronic immunosuppression for prolonged duration to prevent transplant rejection in the transplant recipient [16•]. In solid organ transplant, disease has been described as occurring within 3 months after transplantation with case fatality rates of more than $50 \%$ $[1,3]$. In hematopoietic stem cell transplant, Strongyloides hyperinfection and dissemination has been documented in both autologous and allogenic transplant. Severe cases tend to occur earlier, often immediately post-transplant, compared to solid organ transplant recipients [1]. The difference in symptom onset is thought to be secondary to the acute intense immunosuppression typically associated with stem cell transplant [1]. Stem cell transplant recipients with hyperinfection syndrome and disseminated disease have particularly poor outcomes with one series reporting $85 \%$ mortality [1]. Prognosis in transplanted patients has been shown to improve when steroid therapy can be rapidly tapered [1]. The significant mortality associated with hyperinfection in post-transplant patients highlights the importance of pre-transplant screening [1]. Pretransplant screening guidelines currently recommend screening with Strongyloides-specific IgG via enzymelinked immunosorbent assay (ELISA) for patients from endemic areas or with significant eosinophilia prior to solid organ or hematopoietic stem cell transplant. Patients with positive screening should receive treatment for chronic intestinal strongyloidiasis prior to initiation of immunosuppression [1].

\section{Other IIlnesses Associated with Strongyloidiasis}

Associations between other chronic illnesses and strongyloidiasis have been reported. Patients with severe protein-losing malnutrition and those with hypogammaglobulinemia have increased risk of hyperinfection and disseminated strongyloidiasis. Loss of protein either by primary immunodeficiency or secondary diseases such as in nephrotic syndrome, inflammatory bowel disease, or multiple myeloma have shown increased risk of severe disease and increased risk of refractory disease $[6,14 \bullet, 16 \bullet]$. Case reports of patients with hypogammaglobulinemia had clearance failure of larvae from stool after 14 months of adequate anthelminthic therapy. Such reports suggest that antibodies may play an important role in Strongyloides eradication $[4,18]$. Malignancies including primary organ cancers such as lung and gastrointestinal and primary hematologic malignancies have also been associated with hyperinfection before immunosuppressive chemotherapy and before steroids have been initiated $[3,4,6]$.

\section{Conclusion}

Strongyloides and the host immune system are intimately intertwined. With reduction in cell-mediated host immune response either induced by other infections such as HTLV-1 or through medications such as steroids, chronic strongyloidiasis may have accelerated autoinfection causing hyperinfection syndrome and disseminated disease. Hyperinfection and disseminated strongyloidiasis are associated with high case fatality rates due to burden of disease and due to association with gram-negative bacterial sepsis. Along with increased severity of disease, immunosuppressed patients have increased risk of refractory disease requiring prolonged treatment courses. Improvement in the host immune response through de-escalation of immunosuppression has provided improved outcomes. However, screening prior to initiation of immunosuppression and providing empiric therapy for those at risk may provide the biggest reduction in poor outcomes.

\section{Compliance with Ethics Guidelines}

Conflict of Interest Jill E. Weatherhead and Rojelio Mejia declare that they have no conflict of interest.

Human and Animal Rights and Informed Consent This article does not contain any studies with human or animal subjects performed by any of the authors. 


\section{References}

Papers of particular interest, published recently, have been highlighted as:

- Of importance

1. Roxby AC, Gottlieb GS, Limaye AP. Strongyloidiasis in transplant patients. Clin Infect Dis. 2009;49:1411-23.

2. Levi ME, Kumar D, Green M, Ison MG, Kaul D, Michaels MG, et al. Considerations for screening live kidney donors for Endemic infections: A viewpoint on the UNOS policy. Am J Transplant. 2014;14:1003-11.

3. Montes M, Sawhney C, Barros N. Strongyloides stercoralis: there but not seen. Curr Opin Infect Dis. 2010;23:500-4.

4. Ramanathan R, Nutman TB. Strongyloides stercoralis Infection in the Immunocompromised host. Curr Infect Dis Rep. 2008;10:105-10.

5. Hirata T, Uchima N, Kishimoto K, Zaha O, Kinjo N, Hokama A, et al. Impairment of host immune response against Strongyloides stercoralis by human $\mathrm{t}$ cell lymphotropic virus type I infection. Am J Trop Med Hyg. 2006;74(2):246-9.

6. Keiser PB, Nutman TB. Strongyloides stercoralis in the Immunocompromised population. Clin Microbiol Rev. 2004;17: 208-2017.

7. Porto AF, Neva FA, Bittencourt H, Lisboa W, Thompson R, Alcantara L, et al. HTLV-1 decreases Th2 type of immune response in patients with strongyloidiasis. Parasite Immunol. 2001;23:503-7.

8. Siegel MO, Simon GL. Is Human Immunodeficiency Virus Infection a Risk Factor for Strongyloides stercoralis Hyperinfection and Dissemination? PLOS NTDS. 2012;6:e1581. An excellent review of strongyloidiasis and HIV, history and host immune response.

9. Karp CL, Auwaerter PG. Coinfection with HIV and Tropical Infectious Disease. II. Helminth, Fungal, Bacterial and Viral pathogens. Clin Infect Dis. 2007;45:1214-20.
10. MMWR. Transmission of Strongyloides stercoralis through transplantation of solid organs - Pennsylvania, 2014. 2013; 62(14):264 266.

11. Iriemenam NC, Sanyaolu OU, Oyibo WA, Fagbenro-Beyioku AF. Strongyloides stercoralis and the immune response. Parasitol Int. 2010;59:9-14.

12. Montes M, Sanchez C, Verdonck K, Lake JE, Gonzalez E, Lopez G, et al. Regulatory T Cell expansion in HTLV-1 and strongyloidiasis co-infection is associated with reduced IL-5 responses to Strongyloides stercoralis antigen. PLOS NTDS. 2009;3:e456.

13. Nabha L, Krishnan S, Ramanathan R, Mejia R, Roby G, Sheikh V, et al. Prevalence of Strongyloides stercoralis in an urban US AIDS cohort. Pathog Glob Health. 2012;106:238-44. This research paper addressed the prevalence strongyloidiasis in a US HIV cohort.

14. Le M, Raven K, Hasan A, Clauss H, Muchant DG, Pasko JK, et al. Single Donor Derived Strongyloidiasis in three solid organ transplant recipients: Case series and review of the literature. Am J Transplant. 2014;14:1199-206. An important case series describing risks of strongyloidiasis in solid organ transplant.

15. Salles F, Bacellar A, Amorim M, Orge G, Sundberg M, Lima M, et al. Treatment of strongyloidiasis in HTLV-1 and Strongyloides stercoralis co-infected patients is associated with increased TNF alpha and decreased soluble IL2 receptor levels. Trans R Soc Trop Med Hyg. 2013;107:526-9.

16. Mejia R, Nutman TB. Screening, prevention and treatment for hyperinfection syndrome and disseminated infections caused by Strongyloides stercoralis. Curr Opin Infect Dis. 2012;25:458-63. A comprehensive clinical review of strongyloidiasis and prevention of dissemination.

17. Krishnamurthy R, Dincer HE, Whittemore D. Strongyloides stercoralis Hyperinfection in a Patient with Rheumatoid Arthritis after anti-TNF-alpha therapy. J Clin Rheumatol. 2007;13:150-2.

18. Ashraf M, Cue C, Badour L. Strongyloidiasis Refractory to treatment with ivermectin. Am J Med Sci. 1996;311:178-9. 